\title{
Difficult Male Urethral Catheterization: A Review of Different Approaches
}

\author{
Carlos Villanueva, George P. Hemstreet III
}

Section of Urology, University of Nebraska Medical Center, Omaha, Nebraska, USA

\begin{abstract}
Purpose: To review and compare the different methods for difficult male urethral catheterization described in selected literature.

Materials and Methods: A PubMed search was done with the terms "difficult", "failed", or "complications" and "urethral catheterization", "transurethral catheterization", "Foley catheter", "urethral catheter" or "filiforms and followers". All articles addressing the issue of difficult adult male urethral catheterization were included.

Results: Six main approaches were identified on the 14 articles included for review: 1) Passage of either a Glidewire, guide wire or filiform under direct vision; 2) Blind passage of a filiform, guide wire, Glidewire or hydrophilic catheter; 3) "The Peel-away® sheath placed on a cystoscope/resectoscope technique"; 4) "The rigid ureteroscope placed inside the 22F Foley technique"; 5) Suprapubic catheterization; and 6) "The instillation of $60 \mathrm{cc}$ of saline through the catheter as it is advanced technique".

Conclusion: There is a paucity of prospective data comparing the benefits, risks, success rates and complications of the different approaches for difficult Foley catheter placement. Our suggested approach starts with the initial attempt at urethral catheterization with an $18 \mathrm{~F}$ coude and a $12 \mathrm{~F}$ silicone catheter. If these fail, using a flexible cystoscope or the blind Glidewire technique are reasonable alternatives. If dilatation of a stricture is necessary, ureteric dilatators or a urethral balloon dilatator are recommended.
\end{abstract}

Key words: urethra; male; catheterization

Int Braz J Urol. 2008; 34: 401-12

\section{INTRODUCTION}

Difficult male urethral catheterization(DUC) is a common problem for the general urologist. Common causes of DUC in normal urethras include a tight external sphincter in an anxious patient, or poor technique. Additional pathologic causes include urethral strictures, phimosis, anasarca, bladder neck contractures, prostate cancer, false passages or benign prostatic hypertrophy (BPH), among others (Figure-1). It is underappreciated that a DUC can result in serious morbidity to the patient. The significance of this problem is exemplified by the complications from difficult catheterizations that include Fournier's gangrene (1), rectal perforation, bleeding requiring transfusion, formation of urethral strictures and sepsis.

This article reviews the alternative methods of approaching the difficult-to-catheterize patient described in the literature.

\section{MATERIALS AND METHODS}

A PubMed search was programmed with the terms "difficult", "failed", or "complications" 
and "urethral catheterization", "transurethral catheterization", "Foley catheter", "urethral catheter" or "filiforms and followers" in April of 2008. All articles addressing the issue of difficult adult male urethral catheterization were included. A DUC was defined for the purpose of this article as being unsuccessful at urethral catheterization of the bladder after the initial attempts.

\section{RESULTS}

A summary of the different techniques for urethral catheterization after an unsuccessful attempt is shown in Table-1. Following is a summary of the articles included for review in the chronological order in which they were published. Each paragraph starts with a brief description of the technique. The following information from each article was included when available: risks and benefits of the technique mentioned in the article, complications, success rate, and patient outcomes. All of the statements in each paragraph are derived from the article being discussed.

In 1976, Walden (2) published a technique for DUC in the patient with anasarca using a vaginal speculum. The speculum is passed through the preputial opening down to the glans, and with the use of a long-handled forceps the catheter is advanced into the bladder. He used it in 3 patients, one of whom was a 450-pound ( $220 \mathrm{~kg}$.) man in which the glans was 11 $\mathrm{cm}$ into the swollen prepuce.

Jordan et al. (3) addressed the issue of consultation for DUC in the operating room in 1985. After a through history of previous attempts, past medical history and genitourinary examination, if there are no contraindications to proceed with urethral catheterizations, the first step is the instillation of $20-30 \mathrm{~mL}$ of $2 \%$ lidocaine jelly or other water-soluble lubricating jelly into the urethra. The authors advocated injecting a large volume of jelly for gentle dilation. A 16-18F coude catheter is preferred over a small catheter, which may not be stiff enough to maneuver the difficult urethra. If this is unsuccessful, proceed with either urethroscopy or urethrography. The information gathered from direct or indirect visualization of the urethra would then lead to either suprapubic catheter placement or passage of filiforms. When to proceed with suprapubic catheterization versus the placement of filiforms was not discussed in their article. The passage of a filiform through the point of obstruction should preferentially be done under direct vision. For the blind passage of filiforms it is imperative not to remove the filiform that meets obstruction but to continue placing filiforms until one passes the point of obstruction. When using filiforms and followers it is recommended to dilate only up to $16-18 \mathrm{~F}$ and no larger in order to prevent further damage to the urethra. Finally, a Council tip catheter is advanced over a stylet attached to the filiform. Alternatively, a balloon urethral dilator system that can be passed using a filiform could be used instead. Without supporting data, the authors stated that this balloon system may be better than the use of followers. No specific data regarding success rates or complications was reported in the study.

Krikler (4) described for the first time in 1989 the use of flexible urethroscopy for DUCs. "The cystoscope is negotiated into the bladder, a guidewire is passed through the cystoscope, and the instrument withdrawn leaving the guidewire in place. A ureteric catheter may be passed over the guidewire first and then the tip of a suitable Foley catheter is trimmed to produce an end hole. This allows the catheter to be threaded down the guidewire". He did not make any particular recommendations in the case where a stricture prevented the passage of the flexible cystoscope. This method was recommended for patients in whom suprapubic catheterization is contraindicated or who are known to have false passages or urethral diverticula.

Lowe et al. (5) in 1992 discussed the management of the DUC in patients with multiple false passages, who had just a difficult dilatation with filiforms and followers, in the case of undermining of the bladder neck after trans-urethral resection of the prostate (TURP) or in the early postoperative period after a radical prostatectomy when the catheter came out. The authors described the use of a specially made urethral protective sheath that can be placed on a cystoscope or resectoscope to facilitate guiding the catheter into the bladder. A Peel-Away ${ }^{\circledR}$ Sheath (Cook Urological, Spencer, IN) is placed around the cystoscope or resectoscope at the beginning of the case. Once the 
case is completed, the cystoscope/resectoscope is placed in the bladder and then it is removed leaving the sheath in place. A Foley catheter (with or without a catheter guide) is advanced through the sheath into the bladder, the balloon inflated, and the sheath peeled away. In the case of an unexpectedly difficult cystourethroscopy, once in the bladder, a guide wire can be passed, the cystoscope removed and then reinserted over the wire after placing a Peel-Away ${ }^{\circledR}$ sheath over the scope. The main problem encountered with this technique was kinking of the sheath, which can be prevented by not pulling up or downward on the phallus after the cystoscope has been removed. Kinking of the sheath can also be approached by the use of a catheter guide. This technique was used in 20 difficult catheterizations, with 3 failures, 2 due to kinking of the sheath and one because of the development of an erection making the sheath not long enough to reach the bladder. The authors hypothesized that the Teflon sheaths could be less traumatic to the urethra than the resectoscope sheath and that the use of the sheaths over the resectoscope during a TURP may decrease the post-TURP stricture rate although no data was provided to support this statement.

Cancio et al. (6), in 1993, described a series of initial maneuvers when managing the difficult to catheterize patient. "Start with the injection of 10-20 $\mathrm{mL}$ of lubricant in the urethra and use a $16-18 \mathrm{~F}$ catheter first. The use of $1 \%$ lidocaine jelly as the lubricant makes the procedure more tolerable for the patient and may prevent sphincter spasm. When a stricture is suspected, either because blockage is encountered soon after entering the meatus or because of a history of instrumentation (e.g., TURP), use a smaller catheter (14-16F). If a $14 \mathrm{~F}$ catheter will not pass, a smaller catheter will not pass either. For the patient with suspected $\mathrm{BPH}$, use a larger catheter (20-24F). Perineal pressure by an assistant during catheter insertion can help direct the catheter into the prostatic urethra. Coude catheters were recommended for suspected $\mathrm{BPH}$ and to prevent injury to the membranous and bulbar urethra.

Beaghler et al. (7) method was described in 1994. This method incorporates the use of a $16 \mathrm{~F}$ flexible cystoscope, after injecting $2 \%$ lidocaine jelly, to pass a 0.038 -inch standard guide wire through the obstruction. The urethra is then dilated over the wire with Nottingham dilators $6-12 \mathrm{~F}$ and $12-18 \mathrm{~F}$, and successful catheterization is accomplished by placing a $16 \mathrm{~F}$ council catheter over the wire. This method was attempted in 54 patients prospectively with a success rate of $96 \%$. These patients were seen if urology consultation was requested because of difficulty in placing a Foley or for complaints of weak urinary stream and urinary retention (26\% outpatient clinics, $33 \%$ bedside/Intensive care unit, $13 \%$ emergency room, $28 \%$ operating room). Before attempting the method, catheterization was attempted by standard bedside techniques, including use of various catheters sizes and Coude-tipped catheters. The number of catheters, sizes and types was not specified in the paper, neither was the success rate with these initial maneuvers. The 2 patients in whom this method failed had dense bladder-neck contractures and had a suprapubic catheter placed after a failed attempt at dilating with filiforms and followers. The authors described no complications with little or no discomfort to the patient. The most common causes for difficult Foley catheter placements in this cohort (in order of most to least common) were urethral strictures, bladder-neck contractures, false passages, and locally-advanced prostate cancer.

In 1995, Blitz (8) described a method used in 8 patients that had endoscopic prostate or urethral surgery in which catheters were placed with prior difficulty. With the cystoscope in the bladder, a 0.038 stiff hydrophilic Glidewire is introduced and allowed to coil inside the bladder. Then a " 16 G IV catheter with a needle is passed into the distal drainage hole of the urethral catheter and out through the center of the urethral catheter". The needle is then removed and the wire passed through the catheter. After removing the IV catheter, the wire is directed inside the drainage lumen of the urethral catheter. This maneuver avoided the need for a Council tip catheter, and was better than the alternative of cutting the tip of the Foley with scissors affecting the curvature of the tip of the catheter. A variety of urethral catheters can be used with this method. This approach was successful in all 8 patients. Five patients had just undergone a TURP, 2 patients were status/post direct vision internal urethrotomy, and 1 patient was status/post laser prostatectomy. They stated that their experiences with other wires, including Teflon-coated spiral wound 
Table 1 - Techniques for difficult urethral catheterizations.

\begin{tabular}{lcc} 
Author/Year & Clinical Scenario & Brief Description of the Method \\
\hline $\begin{array}{l}\text { Walden } \\
1979(2)\end{array}$ & $\begin{array}{c}\text { Difficult urethral catheteriza- } \\
\text { tion secondary to anasarca }\end{array}$ & $\begin{array}{c}\text { Use of a vaginal speculum to visualize the glans. Use of a long } \\
\text { forceps to advance the urethral catheter. }\end{array}$
\end{tabular}

Jordan Intraoperative urology con-

et al. 1985 (3) sultation for difficult urethral catheterization

Krikler

1989 (4)

Lowe

et al. $1992(5)$

Cancio

et al. 1993 (6)

Beaghler

et al. 1994 (7)

Blitz

1995 (8)
Traumatized urethra with false channels, post-transurethral resection of prostate undermined bladder neck, and loss of catheter early after radical retropubic prostatectomy.

Initial attempts at urethral catheterization

\section{Urology consultations for} weak urinary stream or patient with prior attempts at catheter placement in the emergency department, operating room, outpatient clinics and intensive care unit/bedside.

Difficult urethral catheterization
Initial use of $20-30 \mathrm{~mL}$ of lidocaine jelly and $16-18 \mathrm{~F}$ coude. Urethrography or urethroscopy to determine if a suprapubic catheter should be placed. Filiforms and followers. Council-type catheter with a stylet attached to the filiform.

Flexible cystoscope negotiated into the bladder. Guide wire advanced through the cystoscope into the bladder and the cystoscope removed. Ureteral catheter advanced over the wire. Foley catheter with the tip trimmed advanced over the guide wire.

Peel-Away ${ }^{\circledR}$ sheath placed on resectoscope or cystoscope. Scope advanced into the bladder. Scope removed leaving sheath in the urethra. Advance Foley through sheath into the bladder. Peelaway the sheath.

Use of 10-20 mL of lidocaine jelly. Large-caliber catheter in patient with benign prostatic hyperplasia (20-24F), small-caliber catheter in patients with stricture (14-16F). Use of coude catheters in males. Use of perineal pressure applied by an assistant.

Advancement of a 0.038 guide wire through the cystoscope under direct vision past the area of obstruction. Sequential dilation with Nottingham dilators, first from 6 to $12 \mathrm{~F}$, then from 12 to $18 \mathrm{~F}$. Placement of 16 F Council-type catheter over the guide wire.

Cystoscope inserted into the bladder. Stiff hydrophilic guide wire passed through the cystoscope into the bladder. Urethral catheter with a hole on the tip made with an IV catheter advanced over the guide wire into the bladder. 
Table 1 - Techniques for difficult urethral catheterizations. - continued -

Freid and Inability to pass a Foley cathSmith eter in cases in which direct 1996 (9) visualization urethroscopy is not immediately available

Harkin One unsuccessful attempt et al. 1998 at urethral catheterization in

Rozanski

et al. 1998

Lachat

et al. 2000

Zammit and German 2004 (13)

Athanassopoulos et al. 2005

Mistry et al. 2007

Chelladurai et al. 2008
Glidewire is advanced into the bladder. 7 Fr open ended ureteral catheter is advanced over the Glidewire and the Glidewire removed. Placement of a 0.038 inch Teflon coated guide wire through the ureteral catheter. $18 \mathrm{~F}$ Graham catheter advanced over the ureteral catheter/guide wire unit. Alternatively, dilate to 16$18 \mathrm{~F}$ followed by the placement of a $16 \mathrm{~F}$ Council-type catheter.

Catheter tip syringe with $60 \mathrm{cc}$ of saline is attached to the Foley catheter. The catheter is introduced into the urethra $2-3 \mathrm{~cm}$ from the point of obstruction and advanced while simultaneously the syringe of fluid is briskly instilled into the urethra.

Short rigid ureteroscope passed through a 22F Foley catheter modified with a catheter punch device. Unit advanced into the bladder under direct vision. Foley secured in place while removing ureteroscope leaving Foley behind.

$30 \mathrm{~cm} 0.035$ inch $\mathrm{J}$ guide wire advance into the bladder. $6 \mathrm{f}-2 \mathrm{~L}$ central line or $6 \mathrm{~F}$ pediatric catheter with the tip cut advanced into the bladder over the wire.

Hydrophilic guide wire advanced into the bladder. 16F Foley with a hole made in the tip with an IV catheter advanced over the guide wire. Alternatively, placement of a $6 \mathrm{~F}$ ureteral catheter over the guide wire, followed by advancement of a graduated 6 to $12 \mathrm{~F}$ semi-rigid ureteral dilator, and then a $12 \mathrm{~F}$ Foley catheter with a hole made on the tip with an IV Catheter.

Straight flexi-tip $0.09 \mathrm{~mm}$ hydrophilic guide wire and a 14/16F ureteric access sheath.

Passage of a $12 \mathrm{~F}$ or $18 \mathrm{~F}$ hydrophilic catheter. Guide wire passed through the catheter into the bladder and the catheter removed. Advance Council-type balloon retention catheter into the bladder.

Negotiate guide wire past stricture under direct vision with a flexible cystoscope. Use serial ureteric dilators over the guide wire. Advance catheter over the guide wire 
guide wires, do not allow such ease of advancement and might cause the catheter to buckle as it is pushed into the bladder. Ex vivo experimentation comparing the Microvasive ${ }^{\circledR}$ Glidewire (Microvasive, Natick, MA) with a Microvasive Lubriglide hydrophiliccoated spiral wound guide wire was nearly equivalent in a subjective evaluation.

Freid and Smith (9) in 1996 described for the first time the use of a 0.038 inch hydrophilic Glidewire (preferentially with an angled or floppy tip) placed blindly into the bladder in a manner similar to a filiform for cases of DUC. The Glidewire is prepared by injecting $5 \mathrm{cc}$ of saline to activate the hydrophilic coating. Lidocaine jelly is then injected in the urethra, followed by the introduction and advancement of the Glidewire with a gentle steady pressure using a gauze pad to grasp it. When resistance is felt, the Glidewire is advanced until either it enters the bladder or the tip appears in the meatus. In the latter situation, the Glidewire is removed and another attempt is made at passing it into the bladder. Entrance into the bladder is inferred by passage of approximately $75 \mathrm{~cm}$ of the Glidewire in the urethra without the reappearance of the tip or a coil at the meatus. Then a 7F ureteral catheter (preferentially with a tapered tip) is threaded over the wire and then, following documented urine return corroborating correct placement, the first wire is exchanged for a 0.038 PTFE coated guidewire. An attempt is made to introduce an $18 \mathrm{~F}$ Graham catheter over the guide wire/ureteral catheter unit, or alternatively the urethra is dilated to $16-18 \mathrm{~F}$ and a $16 \mathrm{~F}$ Council catheter is advanced over the wire. The authors recommended this method over standard filiforms and followers when cystoscopy was not immediately available. This method was used most frequently after failed attempts with filiforms and followers. The reported success rate was 95\% $(19 / 20)$. This method failed in a patient who had a pinhole urethral stricture that necessitated cystourethroscopy with direct vision internal urethrotomy. The authors reported no complications. The most common causes of the difficulty in placing the catheter were, in order of most to least common: urethral strictures, bladder neck contracture, $\mathrm{BPH}$ and unknown. It is unclear how the causes were determined since it was not stated that the patients underwent cystoscopy or other studies.
Harkin et al. (10), in 1998, introduced an entirely different technique that did not involve wires, cystoscopes, IV catheters, or dilators. Their method consists of connecting a catheter tip syringe loaded with $60 \mathrm{~mL}$ of saline to the Foley, inserting the Foley up to where resistance is encountered, and then advancing the Foley while at the same time briskly instilling saline into the distal urethra. It was hypothesized that the flow of fluid distended the urethra facilitating the passage of the catheter, particularly in cases of BPH. The authors recommended using this technique after an unsuccessful attempt at urethral catheterization in the absence of any signs of major urethral trauma. A $100 \%$ success rate was reported in over 30 patients. The causes for the difficulty in catheterizing these patients were not mentioned in the study. They recommended aborting the procedure if severe pain or major resistance were encountered.

Rozanski et al. (11) in 1998 described a technique used in 2 patients with significant undermining of the trigone after transurethral incision of the prostate. In these patients a wire was introduced into the bladder (into a barely visible opening at the 12 o'clock position) and multiple attempts to place a Council catheter over the wire failed. Using a $22 \mathrm{~F}$ Foley with a punch hole at the tip, they inserted a 6 F ureteroscope into the Foley with the tip of the ureteroscope several millimeters beyond the catheter tip, and inserted the ureteroscope and the catheter into the bladder under direct vision. The Foley was grasped securely and the ureteroscope was pulled out. They recommended the use of this technique whenever catheterization is difficult or potentially complicated following transurethral surgery.

Lachat et al. (12) in the year 2000 described a technique for the intraoperative DUC. It consists of advancing a $30 \mathrm{~cm} 0.035$ inch $\mathrm{J}$ guide wire through the urethra into the bladder, followed by either a $6 \mathrm{~F}-$ $2 \mathrm{~L}$ central line or a $6 \mathrm{~F}$ pediatric catheter with the tip cut off advanced over the wire. This method was used with success in 21 patients undergoing cardiovascular surgery in which difficulties with transurethral catheterization were encountered. In 5 patients, 2 or more attempts were required to advance the wire into the bladder. Fluoroscopic guidance was recommended for placement of the wire when a false passage was suspected. 
In 2004, Zammit and German (13) presented a method suggesting the blind passage of a Glidewire (as described by Freid and Smith (9)) as well as the use of an $18 \mathrm{G} \mathrm{IV}$ catheter to perforate the tip of the urethral catheter (as described by Blitz (8)). With this method a $0.89 \mathrm{~mm}$ Terumo Radiofocus ${ }^{\circledR}$ (Terumo Corp., Tokyo, Japan) Guide Wire M Straight is blindly inserted into the bladder by advancing at least $40 \mathrm{~cm}$ of the wire into the urethra (with the urethra being an average of $20 \mathrm{~cm}$, this assures that the wire did not double back on itself). Subsequently an attempt is made to thread a $16 \mathrm{~F}$ urethral catheter over the wire (tip perforation according to the technique described by Blitz (8)). If this is unsuccessful, the possibility of a urethral stricture is considered and a $6 \mathrm{~F}$ ureteral catheter is inserted. If there are no difficulties inserting the ureteral catheter this is removed and a graduated $6 \mathrm{~F}$ to $12 \mathrm{~F}$ semi-rigid ureteric dilator (flexible ureteroscopy introducer) is advanced over the wire into the bladder. This allows the insertion of a $12 \mathrm{~F}$ urethral catheter over the guide wire using the method described by Blitz. The authors recommended aborting the procedure if there were any difficulties when inserting the $6 \mathrm{~F}$ ureteral catheter. The success rate was not reported. However, it was mentioned that patients tolerated the procedure well, and that the cases in which this technique were not successful was due to the creation of a false passage at the initial catheterization attempt. Of note, this method was applied after a failed attempt at urethral catheterization with a $16 \mathrm{~F}$ catheter.

In a letter to Zammit and German (13) Athanassopoulos et al. (14) mentions their unpublished experience using a straight flexi tip hydrophilic Glidewire and a 14/16F ureteric access sheath. They extrapolated the efficacy and atraumatic characteristics of hydrophilic coatings in the ureteric lumen to the urethra. They proposed that "the development of larger diameter hydrophilic sheaths may lead to a totally atraumatic one-step management of urethral strictures".

More recently, Mistry et al. (15) reported in 2007 their experience with the use of hydrophiliccoated urethral catheters in adult males in acute urinary retention with DUC. Criteria for enrollment included a failed attempt at passage of both a $12 \mathrm{~F}$ and $18 \mathrm{~F}$ urethral catheters. Preparation of the hydrophilic catheter consisted of immersion for $5 \mathrm{~min}$ in sterile water and modification of the tip to convert it into a Council-type catheter. An attempt to pass a $12 \mathrm{~F}$ or $18 \mathrm{~F}$ hydrophilic catheter per urethra was made by the urologist. If the hydrophilic catheter successfully passed into the bladder, a guide wire was subsequently passed through the catheter. The hydrophilic catheter was then removed leaving the wire in place, and an attempt to place a standard balloon retention catheter over the guide wire into the bladder was made. If either the hydrophilic catheter did not pass or the balloon retention catheter could not be advanced over the wire, additional intervention was left to the discretion of the physician. Of 44 patients enrolled in the study, $30(68.2 \%)$ underwent successful placement of an indwelling Foley catheter with the use of this method (hydrophilic catheter followed by guide wire followed by balloon retention catheter). The $12 \mathrm{~F}$ hydrophilic catheter was used to gain access into the bladder in 26 patients and an $18 \mathrm{~F}$ hydrophilic catheter was used in 4 patients. The patients, in which this method was not successful, underwent flexible cystoscopy, dilatation with filiforms and followers or Amplatz dilators, and/or blind passage of an open ended catheter with subsequent Foley placement. In the 13 patients that underwent cystoscopy the causes for the difficulty in catheterizing were anterior urethral stricture in 7 , bladder neck contracture in 3 and false passage in 3. They postulated the success of the $12 \mathrm{~F}$ hydrophilic catheter may be related to its increased stiffness when compared to the regular $12 \mathrm{~F}$ latex catheter. In 4 patients, the $12 \mathrm{~F}$ hydrophilic catheter was inserted into the bladder with subsequent failure to pass the balloon retention catheter over the guide wire. In these patients, benefits were still realized (temporary drainage of painful retention, access to the bladder with a guide wire etc.). The authors concluded that incorporating hydrophilic catheters into the urologic armamentarium for catheterizing the difficult urethra will benefit most patients by avoiding more invasive and costly procedures.

Chelladurai et al. (16) recommend using their technique for patients in urinary retention with urethral stricture disease. Under direct vision with a flexible cystoscope, a guide wire is negotiated past the stricture into the bladder. Well lubricated serial ureteric dilators are introduced over the guide wire into 
the bladder. A catheter is then inserted over the guide wire. Delayed definitive treatment of the stricture is undertaken under optimal conditions. They recommend using ureteral over urethral dilators because their narrower caliber, hydrophilic coating and longer length ensure an easier dilatation of the stricture with minimal patient discomfort.

\section{COMMENTS}

The approach to the DUC in the non-trauma setting, where a catheter is needed for urinary retention or to monitor urine output, should start with a through history and physical examination. The history should include, past urologic surgeries (TURP, Radical retropubic prostatectomy), previous difficulties with catheterization, and voiding symptoms, as a clue to the etiology of the problem. Historical information pertaining to previous attempts by nurses or physicians is of utmost importance: the distance at which obstruction was felt $(<16 \mathrm{~cm}$ indicating possible urethral stricture, $>16 \mathrm{~cm}$ indicating $\mathrm{BPH}$, incorrect technique, or bladder neck contracture), whether the Foley balloon was inflated before urine flow (alerting of possible false passage from urethral trauma) or the types and sizes of catheters used, as well as how many people who previously tried (the higher the number that tried could indicate a more difficult catheterization). A focused genitourinary exam may reveal obvious causes for the difficult catheterization penoscrotal edema, phimosis, meatal stenosis, prostate cancer etc.

If a DUC is anticipated based on the above information, we recommend preparing the field by scrubbing the penoscrotal area with an antiseptic solution and placing a $3 / 4$ sheet drape from the scrotum down to the toes. This allows both hands to remain sterile during the procedure to assist in the passage of instruments, and prevents contamination of guide wires, flexible cystoscopes etc. The use of at least 10 cc of a lubricant injected into the urethra should be standard technique. The average volume of the male urethra is $20 \mathrm{~mL}$, suggesting $20 \mathrm{~mL}$ may theoretically be better than $10 \mathrm{~mL}$, but this has not been reported in the setting of DUC. Although most urologists use $2 \%$ viscous lidocaine, its efficacy as an anesthetic was recently questioned in a meta-analysis in which no difference was found when it was compared to plain gel (17). Despite this, patients are familiar with the drug lidocaine and may derive some placebo effect if they know that the drug is being used to numb their urethras. A tense patient usually means a tight urethral sphincter; therefore, patients should be encouraged to relax their legs by pointing their toes outward, while taking slow deep breaths. A firm grasp of the penis with the non-dominant hand, preferentially with gauze around it, pointing at a 45 degree angle is a key element of urethral catheterization. We recommend detaching the catheter from the Foley bag to allow better sensitivity at detecting when passing through the external sphincter and prostate, and to more readily identify obstruction secondary to a false passage or stricture.

The type and size of catheter to be used initially is a detail that has not yet been reported in the setting of DUCs. In a recent online survey (December 2007) of US urology residents at out institution, revealed an $18 \mathrm{~F}$ coude catheter was the first catheter used by the majority of residents for the DUC. With appropriate technique, an $18 \mathrm{~F}$ coude catheter should be advanced with ease into the bladder of the majority of patients in which the difficulty was attributed to an incorrect technique, $\mathrm{BPH}$, or an anxious patient. Only 2 of the articles reviewed mentioned the size / types of catheters employed during the initial attempts at catheterization. In the article by Zammit and German (5), one attempt with a $16 \mathrm{~F}$ urethral catheter was performed before their method was applied. In the Mistry et al. (15) study, patients must have failed urethral catheterization with an $18 \mathrm{~F}$ and a $12 \mathrm{~F}$ urethral catheter as inclusion criteria. As illustrated in Figure-1, there is a high probability that if an $18 \mathrm{~F}$ coude catheter could not be advanced into the bladder, a narrowing of the urethra (urethral stricture, bladder neck contracture) or a false passage is likely the cause of the problem. Since urethral strictures and bladder neck contractures are 5 to 6 times more common than false passages, we recommend another attempt at urethral catheterization with a smaller catheter. Silicone catheters are stiffer than the regular latex catheters and can theoretically provide an advantage when passing a stricture. We use a $12 \mathrm{~F}$ silicone catheter after a failed attempt with an $18 \mathrm{~F}$ coude catheter. The use of more than these 2 


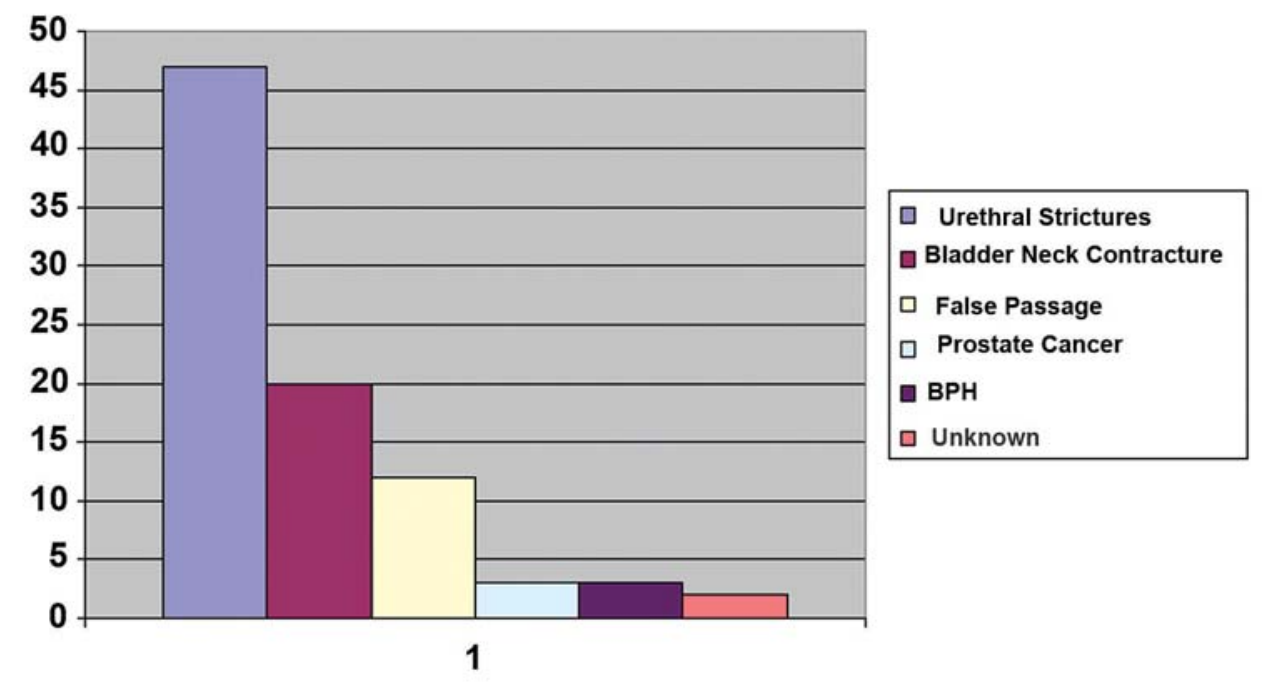

Figure 1 - Most common causes of difficult urethral catheterization*.

* Pooled cases from Beaghler et al. (7), Freid and Smith (9) and Mistry et al. (15) series. Included are the 54 patient from Beaghler's series all of which underwent flexible cystoscopy, and the 13 patient in Mistry et al. series that underwent flexible cystoscopy. Twenty patients from Freid and Smith's series were also included, but it was not mentioned in the article how the cause of difficult urethral catheterization was found in these patients.

catheters, in our opinion, is unlikely to increase the probability of a successful catheterization.

The technique of instilling $60 \mathrm{cc}$ of saline as the catheter is advanced as described by Harkin et al. (10) is a simple one and probably could be attempted before proceeding with any of the other methods if $\mathrm{BPH}$ is suspected. This catheter instillation technique utilizes inexpensive and readily available supplies that can be found on any floor of the hospital or the ER. Although Harkin et al. (10) reported a success rate of $100 \%$ in over 30 patients; these authors do not delineate the causes for the difficulty in placing the Foley. It is intuitive that this method would not be successful in cases of urethral stricture, false passage, or bladder-neck contracture, the most frequent causes of DUC. Therefore, its use is limited to a small proportion of DUCs. Also, proper technique at urethral catheterization requires one hand to handle the phallus and the other to maneuver the catheter, which means that an assistant is required to push the saline. If the catheter is placed in a false passage and saline is forcefully injected, there is a high probability of making the false passage worse. For these reasons we do not advocate for the use of this technique.
Once a patient failed initial attempts at urethral catheterization, the articles reviewed recommend one of the following general approaches: 1) Passage of either a Glidewire, guide wire or filiform under direct vision (with the use of flexible or rigid cystoscopy) past a visible obstruction (i.e. stricture) or into the bladder, followed by the advancement of a modified urethral catheter over the Glidewire, filiform or guide wire, immediately or after dilatation with followers, ureteral dilators etc; 2) Blind passage of a filiform, guide wire, Glidewire or hydrophilic catheter(which is then exchanged for a guide wire) followed by the advancement of a modified urethral catheter over the Glidewire, filiform or guide wire, immediately or after dilatation with followers, ureteral dilators etc; 3 ) "The Peel-away ${ }^{\circledR}$ sheath placed on cystoscope/resectoscope technique"; 4) "The rigid ureteroscope placed inside the 22F Foley technique"; 5) Suprapubic catheterization; and 6) "The instillation of $60 \mathrm{cc}$ of saline through the catheter as it is advanced technique". In our recent online survey of US urology residents to ascertain their approaches to the DUC, $60-70 \%$ used a flexible cystoscope, $15-20 \%$ chose to pass a Glidewire blindly, 7-9\% used filiforms and followers blindly and 
less than 3\% elected a suprapubic catheter after their initial failed attempts at urethral catheterization.

The article by Jordan et al. (3) discusses the blind use of filiforms and followers. They recommend avoiding placing filiforms blindly but also provided recommendations regarding the technique. Freid and Smith (9) described why they stopped using filiforms in favor of their technique. The once popular blind placement of filiforms and followers was chosen only by $7-9 \%$ of urology residents in our recent online survey. Other alternative methods and equipment for dilating strictures like Heymans dilators, balloon dilators and ureteral access sheaths, have contributed to the abandonment of the use of filiforms and followers. To out knowledge there is no reported study comparing the use of filiforms and followers with other techniques used for DUC. Cases of perforation of the urethra and entrance into the rectum with followers are not unheard of. We do not consider the blind use of filiforms and followers to be the optimal approach to the DUC because it is difficult to ascertain if the filiform is in the bladder and requires considerable experience to gain this skill.

Several articles discussed above describe the blind placement of a Glidewire. The main advantage of this approach is that it avoids the cost and inconvenience of the use of a flexible cystoscope. Freid and Smith (9) reported a high success rate with no complications using this method. Insertion of a hydrophilic guide wire though the urethra will usually gain access to the bladder without causing any trauma and with minimal discomfort to the patient. If a Glidewire is placed into a false lumen or cannot transverse a stricture it will usually efface the urethral meatus. If most of the Glidewire has been advanced into the bladder without seeing it coming back at the urethral meatus, there is a high probability that access to the bladder was achieved. If there are still doubts about whether the wire is in the bladder or not, a $6 \mathrm{~F}$ ureteral catheter can be advanced over the wire and urine aspirated or the catheter irrigated with a syringe. Rarely, a bedside plain film of the abdomen is needed to confirm wire placement. There are many types of Glidewires: rigid vs. regular, angled tip vs. straight tip. Freid and Smith's (9) technique used an angled or floppy tip. Zammit and German (13) used a straight tip. Which tip is better to maneuver strictures or a false passage has not been reported. In Mistry et al. (15) article, access to the bladder was gained first with a hydrophilic catheter followed by placement of a guide wire. This technique adds an additional step to the blind placement of a Glidewire. There are probably more chances of being successful at advancing a hydrophilic Glidewire into the bladder than a $12 \mathrm{~F}$ or $18 \mathrm{~F}$ hydrophilic catheter. One of the arguments in favor of using the hydrophilic catheter was that they can readily decompress the bladder in distressed patients in urinary retention. The passage of a Glidewire should not take more than a couple of minutes, and once the wire is in, passing a catheter over the wire should be quick in most situations (as it was in 30 of the 34 of their patients in which access to the bladder was achieved with a hydrophilic catheter in their study).

As the availability of flexible cystoscopes and catheterization carts has become commonplace in the hospital ward, the most frequent approach to the DUC among urology residents in the US is the use of a flexible cystoscope. This approach establishes the etiology of the problem in a majority of cases. In the case of false passages, where occasionally a Glidewire cannot be advanced blindly, the cystoscope can usually be maneuvered into the bladder. Guide wires can also be passed through pinpoint urethral strictures or bladder neck contractures under direct vision. The disadvantages of using this approach are that flexible cystoscopes are not always available, they are expensive, and they usually need to be transported in a cart because of the light source. No study has demonstrated the superiority of the use of flexible cystoscopy in the setting of DUC over other more simple techniques like the blind use of a Glidewire. Still, we believe that flexible cystoscopy is needed in a small percentage of DUCs (mainly in cases of false passages and some urethral strictures) if a suprapubic catheter is to be avoided.

The "Peel-away sheath ${ }^{\circledR}$ placed on cystoscope/resectoscope technique" and "The rigid ureteroscope placed inside the 22F Foley technique" are two maneuvers worth remembering that can be used in specific scenarios but probably cannot be used in the majority of consults for DUC. Urethral catheterization will not always be successful despite the use of all the techniques described and suprapubic catheterization will still be required in rare occasions. 
In conclusion, there are many approaches to the DUC described in the literature. Prospective randomized trials comparing these approaches are needed to determine the strengths and weaknesses of each technique. The paucity of literature related to one of the most common urologic consults was surprising. Finally our recommended approach to the difficult male urethral catheterization in patients with urinary retention or that need a Foley catheter to monitor urine output is an initial attempt with an $18 \mathrm{~F}$ coude catheter followed by a $12 \mathrm{~F}$ silicone catheter. If this approach fails we suggest using the blind Glidewire technique or a flexible cystoscope to pass a Glidewire under direct vision as reasonable options. Once the Glidewire is confirmed to be in the bladder, a $16 \mathrm{~F}$ council catheter can usually be advanced into the bladder. In cases of urethral stricture disease, the primary approach would be to pass a $12 \mathrm{~F}$ silicone catheter (using Blitz (8) technique) over the Glidewire without dilating, with a delayed definitive treatment of the stricture under optimal conditions. In cases of tight strictures in which a $12 \mathrm{~F}$ did not pass or when treatment of the stricture is desired, serial ureteric dilators or a urethral balloon dilatator passed over the Glidewire are reasonable alternatives.

\section{CONFLICT OF INTEREST}

\author{
None declared.
}

\section{REFERENCES}

1. López Pacios JC, Sánchez Merino JM, Piñeiro Fernández MC, Bouso Montero M, Parra Muntaner L, García Alonso J. Fournier's gangrene secondary to urethral catheterization. Arch Esp Urol. 2005; 58: 167-70.

2. Walden TB: Urethral catheterization in anasarca. Urology. 1979; 13: 82.

3. Jordan GH, Winslow BH, Devine CJ Jr: Intraoperative consultation for the urethra. Urol Clin North Am. 1985; 12: 447-52.

4. Krikler SJ: Flexible urethroscopy: use in difficult male catheterisation. Ann R Coll Surg Engl. 1989; 71: 3.

5. Lowe MA, Defalco AJ: New endourologic technique for catheter placement after TURP, prostatectomy, and difficult urethroscopy. Urology. 1992; 40: 461-3.
6. Cancio LC, Sabanegh ES Jr, Thompson IM: Managing the Foley catheter. Am Fam Physician. 1993; 48: 829-36.

7. Beaghler M, Grasso M 3rd, Loisides P: Inability to pass a urethral catheter: the bedside role of the flexible cystoscope. Urology. 1994; 44: 268-70.

8. Blitz BF: A simple method using hydrophilic guide wires for the difficult urethral catheterization. Urology. 1995; 46: 99-100.

9. Freid RM, Smith AD: The Glidewire technique for overcoming urethral obstruction. J Urol. 1996; 156: 164-5.

10. Harkin DW, Hawe M, Pyper P: A novel technique for difficult male urethral catheterization. Br J Urol. 1998; 82: 752-3.

11. Rozanski TA, Salazar F, Thompson IM: Direct vision bladder catheterization using a short rigid ureteroscope. Urology. 1998; 51: 827-8.

12. Lachat ML, Moehrlen U, Bruetsch HP, Vogt PR: The Seldinger technique for difficult transurethral catheterization: a gentle alternative to suprapubic puncture. $\mathrm{Br}$ J Surg. 2000; 87: 1729-30.

13. Zammit PA, German K: The difficult urethral catheterization: use of a hydrophilic guidewire. BJU Int. 2004; 93: 883-4.

14. Athanassopoulos A, Liatsikos EN, Barbalias GA: The difficult urethral catheterization: use of a hydrophilic guidewire. BJU Int. 2005; 95: 192.

15. Mistry S, Goldfarb D, Roth DR: Use of hydrophiliccoated urethral catheters in management of acute urinary retention. Urology. 2007; 70: 25-7.

16. Chelladurai AJ, Srirangam SJ, Blades RA: A novel technique to aid urethral catheterisation in patients presenting with acute urinary retention due to urethral stricture disease. Ann R Coll Surg Engl. 2008; 90: 77-8.

17. Patel AR, Jones JS, Babineau D: Lidocaine $2 \%$ gel versus plain lubricating gel for pain reduction during flexible cystoscopy: a meta-analysis of prospective, randomized, controlled trials. J Urol. 2008; 179: 986-90.

Accepted after revision:

June 1, 2008

\footnotetext{
Correspondence address:

Dr. Carlos Villanueva

Section of Urologic Surgery

University of Nebraska Medical Center

855, N 82ND Plaza Apt 40

Omaha NE 68114, USA

Fax: + 1402 990-3634

E-mail: cvillanu@unmc.edu
} 


\section{EDITORIAL COMMENT}

In this manuscript, the authors present a review of the literature on the management of difficult male catheterization. Using a limited PubMed search, they retrieved 6 papers on the topic. It is an "information only" type of paper, as it does not provide direct recommendations for clinical practice. However, the various methods proposed are interesting to the reader as well as the creative solutions surgeons have tried in the management of what is a huge problem for both the patient and the care staff.

\author{
Dr. Katherine N. Moore \\ University of Alberta \\ Faculty of Nursing \\ Edmonton, Alberta, Canada \\ E-mail: katherine.moore@ualberta.ca
}

\section{EDITORIAL COMMENT}

Difficult male urethral catheterization is still a common problem for the general urologist. It can be a challenge for different reasons, such as urethral stricture, benign prostatic hypertrophy or cancer. It must be reminded that bladder neck contractures and obliterated anastomosis are still present following transurethral resection of the prostate, supra-pubic prostatectomy and radical prostatectomy, in spite of all attempts to avoid them. Unfortunately, the common scenario of "difficult" urethral catheterization after multiple attempts of non-urological stuff is still a part of our daily practice. Sometimes these "emergent calls" come from the emergency or operation room. These circumstances put our young colleagues in a stressful situation making their task more difficult. That is why it is particularly important to construct an algorithm for these situations.

A lot of possible techniques for difficult urethral catheterization have been described (1-4), however there are still no guidelines for practical purposes. The authors of this manuscript not only describe these methods, but also suggest which of them could be use in special situation. They must be encouraged for this attempt to construct a kind of guidelines for difficult urethral catheterization. I hope that this manuscript will be very popular among residents.

\section{REFERENCES}

1. Chelladurai AJ, Srirangam SJ, Blades RA: A novel technique to aid urethral catheterisation in patients presenting with acute urinary retention due to urethral stricture disease. Ann R Coll Surg Engl. 2008; 90: 77-8.

2. Carr LK, Webster GD: Endoscopic management of the obliterated anastomosis following radical prostatectomy. J Urol. 1996; 156: 70-2.

3. Chiang DT, Dewan PA, Ben-Meir D: The difficult urethral catheterization: use of a hydrophilic guidewire. BJU Int. 2004; 94: 447-8.

4. Lachat ML, Moehrlen U, Bruetsch HP, Vogt PR: The Seldinger technique for difficult transurethral catheterization: a gentle alternative to suprapubic puncture. $\mathrm{Br}$ J Surg. 2000; 87: 1729-30.

Dr. Sergey Kravchick Department of Urology Barzilai Medical Center Ashkelon, Israel E-mail:cambell@netvision.net.il 